\title{
Reaching the Limits of Nuclear Existence
}

\section{Researchers have identified the largest possible isotopes of fluorine and neon, extending the neutron "dripline" for the first time in 20 years.}

\section{by Artemis Spyrou*}

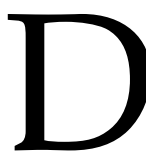
iscovering nature's limits has always been a major goal of physics research. For rare isotopes, this quest takes the form of finding the combinations of protons and neutrons that lead to a bound nuclear system. For a nucleus with a set number of protons, there is a limit to how many neutrons can be added before they stop sticking together and instead drip out. This limit, known as the neutron dripline, represents the border between the bound and unbound isotopes of a particular element. Now, for the first time in 20 years, researchers have extended the neutron dripline, locating the boundary for two elements heavier than oxygen. Deuk Soon Ahn at RIKEN, Japan, and colleagues determined the maximum number of neutrons that fluorine and neon can have, setting new constraints for theoretical calculations [1].

Previously, the neutron dripline had been measured for only the eight lightest of the 118 known elements [2]. Map-

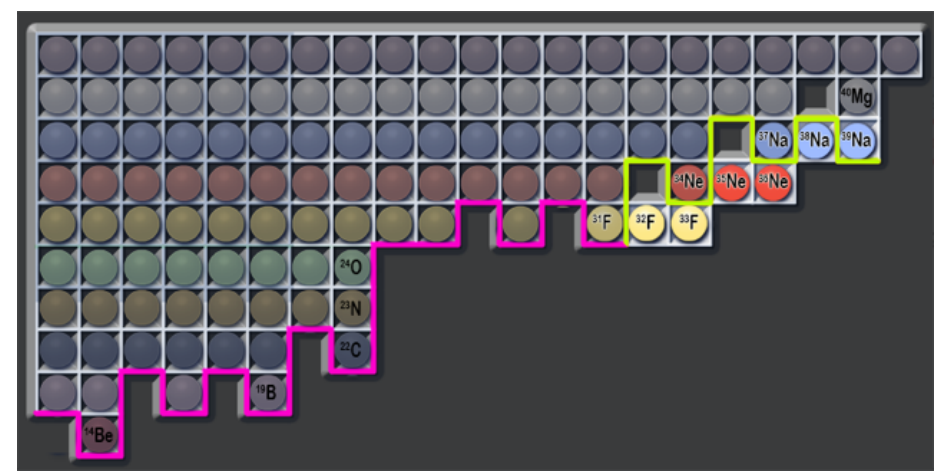

Figure 1: Researchers have mapped the boundary (green line) that charts the heaviest possible isotopes of fluorine $(F)$ and neon $(\mathrm{Ne})$. Previously this so-called neutron dripline was known only for the first eight elements of the periodic table (pink line). (APS/ Joan Tycko)

*Department of Physics and Astronomy, Michigan State University, East Lansing, MI 48824, USA ping the neutron dripline for heavier elements allows scientists to better understand the limits of nuclear existence. But such experiments do more than plot the edges of the chart of nuclides; they have the potential to challenge what we know about the fundamental forces of nature that define the structure of these exotic nuclei. For example, experiments have shown that 16 is a "magic" number for neutrons $(N)$ in very neutron-rich nuclei, meaning that isotopes with $N=16$ are unusually stable [3]. Researchers have linked this observation to the location of the dripline for carbon, nitrogen, and oxygen, all of which have 16 neutrons in their heaviest bound isotopes [4]. But further experiments are needed to fully understand the "magicity" of $N=16$ and its dripline connection.

These experiments could also help explain the unexpectedly small $N$ of oxygen's heaviest isotope. While fluorine can bind at least 22 neutrons $\left({ }^{31} \mathrm{~F}\right)$, oxygen-the preceding element in the periodic table-has no isotope with more than 16 neutrons $\left({ }^{24} \mathrm{O}\right)$. Most theoretical models fail to reproduce this observed feature, known as the "oxygen anomaly," predicting instead that ${ }^{26} \mathrm{O}$ or even ${ }^{28} \mathrm{O}$ should be bound. The oxygen anomaly has prompted many interpretations, stretching our understanding of the nature of the nuclear force. For example, some theorists posit that its cause is repulsive contributions to the force that arises when three neutrons interact, as the attractive forces between neutron pairs are insufficient to explain oxygen's behavior [5]. On the other hand, other researchers propose that the added stability of fluorine isotopes is due to the emergence of an "island of inversion" [6] - a region on the chart of nuclides where patterns of behavior predicted by the traditional nuclear shell model do not hold. These ideas notwithstanding, we still lack a complete physical model of exotic neutronrich isotopes, so the dripline locations of two more elements represent critical new observations that offer new clues for theoretical models to follow.

In the new measurement, Ahn and colleagues fired a highenergy beam of ${ }^{48} \mathrm{Ca}$ at a beryllium target. When the beam hit the target, the calcium nuclei broke into pieces in a nuclear reaction called fragmentation. The team then looked for exotic isotopes using a powerful "filter" - the fragmentseparator BigRIPS, which discards unwanted species and 
isolates the isotopes of interest based on their mass and charge. With the dripline already plotted for the elements up to oxygen, the researchers looked specifically for isotopes of fluorine, neon, and sodium - the next elements in the periodic table-whose heaviest known isotopes are ${ }^{31} \mathrm{~F},{ }^{34} \mathrm{Ne}$, and ${ }^{37} \mathrm{Na}$. The dripline traces an irregular path through the table of nuclides, sometimes excluding one isotope while including its heavier neighbor. Ahn and colleagues therefore sought to pin down the boundary by searching for the next two isotopes of each element- ${ }^{32,33} \mathrm{~F},{ }^{35,36} \mathrm{Ne}$ and ${ }^{38,39} \mathrm{Na}$.

The team observed no events for ${ }^{32,33} \mathrm{~F},{ }^{35,36} \mathrm{Ne}$ and ${ }^{38} \mathrm{Na}$, but given the sensitivity of the measurements, they should have detected between 5 nuclei $\left({ }^{38} \mathrm{Na}\right)$ and 1100 nuclei $\left({ }^{32} \mathrm{~F}\right)$ if these isotopes were bound. The failure to spot them let the researchers place sensitive confidence limits on these isotopes' nonexistence: the chances that they are bound are one in a hundred for ${ }^{38} \mathrm{Na}$ and less than one in ten billion for the fluorine isotopes. From these measurements, they established that the last bound isotope of fluorine is ${ }^{31} \mathrm{~F}$ with 22 neutrons, and the heaviest isotope of neon is ${ }^{34} \mathrm{Ne}$, with 24 neutrons. However, the researchers did observe one event for ${ }^{39} \mathrm{Na}$, indicating that this isotope is most likely bound, and that the dripline for sodium lies somewhere beyond this point.

The new measurements by Ahn and colleagues present a significant challenge to state-of-the-art theoretical calculations, which currently reproduce the observations for only one of these elements. Specifically, while some models predict that the dripline should be at $N=24$ for both fluorine and neon [4], Ahn and colleagues found that fluorine's last bound isotope has $N=22$. These models will therefore need to be revised to accommodate the experimental results, an action that could shed light on fundamental properties of nuclei, such as how nucleons interact under extremely neutron-rich conditions.
The exciting new results of Ahn and colleagues mark an important step forward in rare isotope science that took 20 years to take. Reaching the neutron dripline for even heavier elements is a major goal of the field, and there's a good chance that we won't have to wait so long for the next discovery. While dripline research continues at RIKEN, new measurements are being planned at next-generation rareisotope facilities around the world, like the Facility for Rare Isotope Beams (FRIB) in the US. FRIB, which is expected to be completed in about two years, will have beams that are significantly more intense than RIKEN's and thus could reach the dripline for up to magnesium, the 12th element of the periodic table, in the next five years or so. Combined with modern theoretical models, these new measurements pave the way for a better understanding of the atomic nucleus at the extremes.

This research is published in Physical Review Letters.

\section{REFERENCES}

[1] D. S. Ahn et al., "Location of the neutron drip line at fluorine and neon," Phys. Rev. Lett. 123, 212501 (2019).

[2] M. Thoennessen, "Current status and future potential of nuclide discoveries," Rep. Prog. Phys. 76, 056301 (2013).

[3] C. R. Hoffman et al., "Evidence for a doubly magic ${ }^{24} \mathrm{O}$," Phys. Lett. B 672, 17 (2009).

[4] I. Tanihata, D. Hirata, and H. Toki, "Are all nucleus spherical at the drip line?" Nucl. Phys. A 583, 769 (1995).

[5] T. Otsuka, T. Suzuki, J. D. Holt, A. Schwenk, and Y. Akaishi, "Three-body forces and the limit of oxygen isotopes," Phys. Rev. Lett. 105, 032501 (2010).

[6] E. K. Warburton, J. A. Becker, and B. A. Brown, "Mass systematics for $A=29-44$ nuclei: The deformed $A \sim 32$ region," Phys. Rev. C 41, 1147 (1990).

10.1103/Physics.12.126 